\title{
Genetic Diversity among U.S. Garlic Clones as Detected Using AFLP Methods
}

\author{
Gayle M. Volk¹, Adam D. Henk, and Christopher M. Richards \\ National Center for Genetic Resources Preservation, U.S. Department of Agriculture, Fort Collins, \\ CO 80521
}

AdDitional INDEX wORDs. Allium spp., molecular marker, duplicate accessions

\begin{abstract}
Garlic (Allium sativum L.) has been clonally propagated for thousands of years because it does not produce seed under standard cultivation conditions. A single garlic accession frequently displays a high degree of phenotypic plasticity that is likely to be dependent upon soil type, moisture, latitude, altitude, and cultural practices. The diversity observed by collectors has occasionally led to the renaming of varieties as they are exchanged among growers and gardeners. As a result, there are numerous garlic varieties available both commercially and within the USDA National Plant Germplasm System (NPGS) that may be identical genotypically, yet have unique cultivar names. To address this possibility, we performed amplified fragment-length polymorphism (AFLP) analysis on a comprehensive selection of 211 Allium sativum and Allium longicuspis accessions from NPGS and commercial sources. We used several statistical approaches to evaluate how these clonal lineages are genetically differentiated and how these patterns of differentiation correspond to recognized phenotypic classifications. These data suggest that while there are extensive duplications within the surveyed accessions, parsimony and distance based analyses reveal substantial diversity that is largely consistent with major phenotypic classes.
\end{abstract}

Garlic (Allium sativum L.) is a vegetatively propagated crop that does not set seed under standard growing conditions. Clonal lineages within this species show a remarkably high degree of phenotypic diversity. New genotypes have not been obtained through hybridization, but through the selection of spontaneous mutations expressing traits of horticultural interest. More than 200 named garlic clones are commercially available in the United States. The U.S. National Plant Germplasm System (NPGS) maintains 193 main accessions (with PI, Plant Introduction, and W6 prefixes) at the Western Regional Plant Introduction Station (WRPIS) in Pullman, Wash. The diversity of these clones is described by a set of phenotypic and morphological descriptors known to be phenotypic and morphological descriptors known to be plastic (Al-Zahim et al., 1997; Ipek et al., 2003). Plant and bulb characteristics are determined by growing season, winter conditions, location, as well as nutrient and water availability. Most likely, many of the commercial garlic cultivars have been renamed as they have been traded among growers and gardeners for hundreds of years.

Maintenance of these accessions requires a substantial effort since all clones must be planted in the field each fall and bulbs are harvested the following summer. The collection is at risk because pathogens may easily invade field plots and the shelf life of most accessions is less than a year. Methods are being developed to backup the NPGS garlic collection cryogenically. Cryopreservation is time-consuming, expensive, and often genotype dependent. However, when reliable methods are developed, long-term preservation can be attained (Cho et al., 2003; Keller, 2002; Makowska et al., 1999).

Garlic has been cultivated for millennia, but the taxonomic origins of this domestication process have not been identified. Allium

Received for publication 10 Dec. 2003. Accepted for publication 27 Feb. 2004. We thank Walter Lyons, Barbara Hellier, and Tom Cloud for providing garlic samples and assisting with cultivar identification. We appreciate the technical assistance provided by Jackie Harris and Pat Reeves. Mention of trade names or commercial products in this article is solely for the purpose of providing specific information and does not imply recommendation or endorsement by the U.S. Department of Agriculture.

${ }^{1}$ Corresponding author; e-mail gvolk@lamar.colostate.edu. longicuspis, which can be found in the wild in Central Asia, was once thought to be the living progenitor of A. sativum. However, recent molecular evidence indicates that $A$. longicuspis lies within the range of genetic diversity found within A. sativum (Etoh and Simon, 2002). Many classification systems for garlic have been described (Etoh and Simon, 2002). Most systems describe subspecies that are either bolting types or non and incomplete bolting types and are termed ophioscorodon and sativum, respectively (Etoh and Simon, 2002). Garlic growers have further classified garlic varieties based on phenotypic characters as exhibited in Okanogan, Wash. (Engeland, 1991, 1995) (Table 1). Since most commercially available garlic cultivars in the United States have been classified according to this scheme, we used this system to associate morphological features with our genetic analyses. Table 1 includes a column to convert the classification system proposed by Engeland $(1991,1995)$ to the subgroup classification system used in Germany and described by Keller (2002), Hanelt (2001), and Maass and Klaas (1995).

Allium sativum ssp. sativum includes the softneck garlic cultivars that are either nonbolting or produce only very weak stalks. In general, most softneck types have 12 to 20 cloves arranged in three to six layers within a bulb. Artichoke-type softnecks tend to have early maturing cloves with coarse, thick clove skins and yellow-green, horizontal leaves that emerge from all sides of the plant. During stressful conditions, asexual propagules called bulbils may be produced within the false stems, resulting in the classification of incomplete bolting types. In contrast, silverskintype softnecks have nearly vertical, blue-green leaves that display a bilateral symmetry. The tight clove skin on silverskins and late maturation dates enable these types to be stored for long periods of time. Softneck cultivars often grow best under mild winter conditions (Engeland, 1991, 1995).

Allium sativum ssp. ophioscorodon includes the hardneck garlic types that produce scapes, or flower stalks. These types tend to prefer northerly U.S. climates with cold winters. Many of these types produce a single layer of cloves around the stalk within the bulb. Rocambole types exhibit one to three coils in the developing scape and have 6 to 11 rounded cloves. Bulbs store poorly since cloves have loose, easily peeled clove skins. 
Table 1. Phenotypic types of garlic as described by Engeland et al. (1991, 1995).

\begin{tabular}{|c|c|c|c|c|c|c|c|c|}
\hline Type & $\begin{array}{c}\text { Scape } \\
\text { curl }\end{array}$ & $\begin{array}{l}\text { Clove } \\
\text { skin }\end{array}$ & Bulbils & $\begin{array}{c}\text { Leaf } \\
\text { architecture }\end{array}$ & $\begin{array}{l}\text { Leaf } \\
\text { color }\end{array}$ & $\begin{array}{c}\text { Clove } \\
\text { no. }\end{array}$ & $\begin{array}{c}\text { Clove } \\
\text { arrangement }\end{array}$ & $\begin{array}{c}\text { Corresponding } \\
\text { subgroup }^{z}\end{array}$ \\
\hline Silverskin & none & tight & none & nearly vertical & blue-green & $8-24$ & 3-6 layers & sativum \\
\hline Artichoke & none & coarse and thick & none or lower stem & nearly horizontal & yellow-green & $12-16$ & 3-6 layers & sativum \\
\hline Rocambole & $1-3$ coils & easily peeled & 10-40, br, y, purple & & & $3-14$ & single layer & ophioscorodon \\
\hline Porcelain & random & tight & many tiny white & & & $2-5$ & single layer & sativum \\
\hline Purple Stripe & 0.75 loop & easily peeled & $>50$ purple & & & $8-12$ & single layer & longicuspis \\
\hline Marble purple stripe & 0.75 loop & easily peeled & 20-60 purple & & & $4-7$ & single layer & longicuspis \\
\hline Asiatic & droops & tight & few, purple & & & $4-8$ & single layer & pekinse \\
\hline Turban & U downwards & coarse and thick & 30-100 small purple & & & $7-11$ & single layer & \\
\hline Creole & U downwards & tight & $>30$ small-medium & & & $4-8$ & single layer & \\
\hline
\end{tabular}

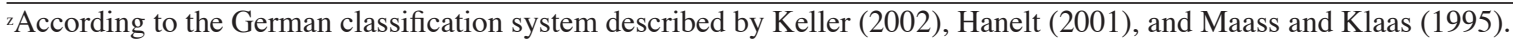

Rocamboles generally produce 10 to 40 brown, yellow, or purple bulbils in the umbels. A former classification included a category titled "continental"; varieties that had many tiny bulbils. This category has now been divided into the purple stripe (including purple stripes and marble purple stripes) and porcelain variety types. Both purple stripe and marble purple stripe garlic types have scapes that make a $270^{\circ}$ loop and produce cloves that can be easily peeled. Purple stripe garlics have 8 to 12 tallish and crescent-shaped cloves, while marble purple stripe garlics have 4 to 7 rounded cloves per bulb. Porcelain garlic types have scapes that have random curling characteristics, tight clove skins, and four to six elongated cloves (Engeland, 1991, 1995).

There is some confusion with respect to the classification of the Asiatic, Creole, and Turban garlic types. Genetic analyses of Pooler and Simon (1993) classify these garlic types as softnecks, despite the fact that they occasionally make scapes. Asiatic garlic types have drooping scapes and six to nine plump, glossy skinned cloves per bulb with a few purple bulbils in an elongated, wrinkled bulbil capsule (Engeland, 1991, 1995). Turban-type garlics are similar to Asiatics, with a turban-shaped bulbil capsule and a very early maturation date, often sprouting by the October planting date. Creoles have vivid wrappers, tallish cloves with elongated tips and numerous tiny bulbils, and grow best in the southern United States. Creole scapes are generally short and weak (Engeland, 1991, 1995).

Most of the commercial garlic cultivars have been classified into one of the categories listed above. However, this sort of phenotypic classification is not available for most accessions maintained in the NPGS.

The purpose of this study is to examine the diversity of a collection of 211 commercial and federally held accessions using a set of amplified fragment-length polymorphism (AFLP) markers. Analysis of this data was subjected to several analytical techniques that allowed us to compare the molecular diversity of these accessions a phenotypic classification scheme. By including both commercial and federal collections in our dataset, we can identify both the structure of diversity and the extent of duplications within the collection. In addition, these data will be valuable in prioritizing representative accessions for further cryopreservation research.

\section{Materials and Methods}

Plant material for analysis. Garlic cloves were provided by the WRPIS (Pullman, Wash.) and from commercial growers Walter Lyons (www.thegarlicstore.com) and Tom Cloud (www. filareefarms.com). Accession data are provided in Table 2. In the collection of 211 accessions in this data set, 20 named cultivars were represented by 47 clones. These represent accessions that had the same cultivar name but were obtained from different sources. Accessions duplicated in this manner are identified in Table 2 with an appended number to the name, e.g., 'Siberian-1' and 'Siberian2'. Having these duplications allowed us to test whether cultivars bearing the same name but collected from different growers are genetically identical. Garlic cultivars were classified by the growers. NPGS accessions were classified as hardneck or softneck by identifying a stem in cross-sectional images of garlic accessions publicly available through GRIN (Germplasm Resources Information Network, http://www.ars-grin.gov/npgs/).

Molecular analysis. DNA was independently extracted from shoot tips within two cloves from each accession using the DNeasy 96 Plant Kit (Qiagen, Valencia, Calif.) with some minor modifications. For each extraction, $50 \mathrm{mg}$ of shoot tip tissue was frozen in liquid nitrogen and ground to a powder. Initial extraction volumes using the lysis buffer AP1 was doubled to $800 \mu \mathrm{L}$ per individual. AFLP technique (Vos et al., 1995) was performed on each DNA sample as described by Myburg et al. (2000). In short, $500 \mathrm{ng}$ of genomic DNA was digested with EcoRI and MseI restriction enzymes (New England Biolabs, Beverly, Mass., and Invitrogen, Carlsbad, Calif.). Adapters (Table 3) were ligated to the digested genomic DNA. The ligation products were diluted 1:10 with distilled water and preselective PCR amplification was performed using an MJ Research (Reno, Nev.) PTC200 or Dyad thermalcycler. Products were diluted 1:40 with low TE [0.1 mM EDTA, 10 mm Tris (Sigma-Aldrich Corp., St. Louis), pH 8.0] and selectively amplified using one of the primer sets listed in Table 3. Adapters and nonlabeled primers were synthesized by IDT (Skokie Ill.) and dye-labeled primers were synthesized by MWG Biotech (High Point, N.C.). Labeled products were separated with a LI-4200 DNA sequencer (LI-COR, Lincoln, Neb.) and digitally captured using the Saga 2.0 software package (LI-COR).

Data ANALYSIS. TIFF images were retrieved and partially analyzed using SAGA 2.0 MX module (LI-COR). We manually selected the most informative and consistently reproducible markers for analysis in this data set. These 27 loci were manually scored for each of the two independent replicate samples from each accession. When one of the 27 loci was different among the replicate samples, the locus was omitted as unreconciled and a missing (ambiguous) data point replaced this locus in the consensus score. Samples with $>4 \%$ (more than one ambiguous value for 27 loci scored) were removed from the dataset and not included in any analyses. In the data for this study, there were a total of 211 samples. Of these samples, 158 samples had a complete genotype at all 27 loci and 53 had one ambiguous value. These genotypes represent intraspecific diversity and as such were analyzed in a way to display the most conservative clustering. We used principle 
Table 2. Names, phenotypic classification, and genetic classification of garlic clones.

\begin{tabular}{|c|c|c|c|c|c|c|}
\hline Cultivar identifier & WRPIS cultivar & Source & Type & Class & PCA & Group \\
\hline W6-49 & M89-39 & WRPIS & Hardneck & Hardneck & $\mathrm{I}$ & $\mathrm{A}$ \\
\hline W6-671 & W6-671 & WRPIS & Softneck & Softneck & I & $\mathrm{D}$ \\
\hline W6-672 & W6-672 & WRPIS & Softneck & Softneck & & $\mathrm{D}$ \\
\hline W6-1861 & Dunganski & WRPIS & Hardneck & Marble PS & IV & \\
\hline W6-1862 & Ferganski & WRPIS & Hardneck & Purple Stripe & I & $\mathrm{H}$ \\
\hline W6-1880 & longicuspis & WRPIS & Hardneck & longicuspis & & \\
\hline W6-1883 & Maiski & WRPIS & Hardneck & Asiatic & I & $\mathrm{H}$ \\
\hline W6-1885 & Ferganski & WRPIS & Hardneck & Purple Stripe & IV & $\mathrm{L}$ \\
\hline W6-1903 & longicuspis & WRPIS & Hardneck & longicuspis & IV & \\
\hline W6-2560 & W6-2560 & WRPIS & Softneck & Softneck & I & $\mathrm{D}$ \\
\hline W6-2561 & Arguni White & WRPIS & Softneck & Softneck & & $\mathrm{D}$ \\
\hline W6-2562 & Arguni White & WRPIS & Softneck & Softneck & I & $\mathrm{D}$ \\
\hline W6-2563 & W6-2563 & WRPIS & Softneck & Softneck & I & $\mathrm{D}$ \\
\hline W6-8403 & Aja rondena & WRPIS & Softneck & Softneck & I & $\mathrm{D}$ \\
\hline W6-8404 & Bhsto de chinchon & WRPIS & Softneck & Softneck & & $\mathrm{D}$ \\
\hline W6-8405 & Blanco de bonda & WRPIS & Softneck & Softneck & I & $\mathrm{D}$ \\
\hline W6-8406 & Blanco de clenla & WRPIS & Softneck & Softneck & I & $\mathrm{D}$ \\
\hline W6-8409 & Blanco de valla dolid & WRPIS & Softneck & Softneck & & $\mathrm{D}$ \\
\hline W6-8410 & Blanco gallego & WRPIS & Softneck & Softneck & I & $\mathrm{D}$ \\
\hline W6-8411 & Blanco vallelado & WRPIS & Softneck & Softneck & I & $\mathrm{D}$ \\
\hline W6-8413 & Labera del obispo & WRPIS & Hardneck & Hardneck & I & A \\
\hline W6-8414 & Manuel benitee & WRPIS & Hardneck & Hardneck & & Q \\
\hline W6-8415 & Morado de pedronera & WRPIS & Hardneck & Hardneck & I & A \\
\hline W6-8416 & Rojo de banolas & WRPIS & Hardneck & Hardneck & I & Q \\
\hline W6-8417 & Rojo de castro & WRPIS & Hardneck & Hardneck & & A \\
\hline W6-8420 & Rojo del Pais baza & WRPIS & Hardneck & Hardneck & & A \\
\hline W6-10473 & E92-16 & WRPIS & Hardneck & Hardneck & I & $\mathrm{H}$ \\
\hline W6-10729 & B92-16 & WRPIS & Softneck & Softneck & I & $\mathrm{D}$ \\
\hline W6-10734 & B92-21 & WRPIS & Softneck & Softneck & & $\mathrm{D}$ \\
\hline W6-10736 & B92-23 & WRPIS & Softneck & Softneck & I & D \\
\hline W6-10737 & B92-24 & WRPIS & Softneck & Softneck & & $\mathrm{D}$ \\
\hline W6-10739 & B92-222 & WRPIS & Softneck & Softneck & I & $\mathrm{D}$ \\
\hline W6-12816 & WWE Italian garlic & WRPIS & Softneck & Silverskin & I & $\mathrm{D}$ \\
\hline W6-12823 & $199-33$ & WRPIS & Hardneck & Hardneck & & $\mathrm{O}$ \\
\hline W6-12824 & $199-36$ & WRPIS & Hardneck & Hardneck & II & $\mathrm{F}$ \\
\hline W6-12825 & $199-41$ & WRPIS & Hardneck & Hardneck & & $\mathrm{F}$ \\
\hline W6-12828 & $850910-2$ & WRPIS & Hardneck & Hardneck & II & $\mathrm{F}$ \\
\hline W6-12829 & $851004-1$ & WRPIS & Hardneck & Hardneck & II & $\mathrm{F}$ \\
\hline W6-12830 & $8510301-1$ & WRPIS & Softneck & Softneck & I & A \\
\hline W6-12831 & $870529-01$ & WRPIS & Hardneck & Hardneck & & A \\
\hline W6-12832 & 870825 & WRPIS & Hardneck & Hardneck & I & A \\
\hline W6-12833 & 871210 & WRPIS & Hardneck & Hardneck & & $\mathrm{F}$ \\
\hline W6-12836 & French garlic & WRPIS & Softneck & Softneck & I & $\mathrm{D}$ \\
\hline W6-12837 & GSF-18 & WRPIS & Hardneck & Hardneck & II & $\mathrm{F}$ \\
\hline W6-12838 & GSF-60 & WRPIS & Hardneck & Hardneck & II & F \\
\hline W6-12839 & Gourmet Red & WRPIS & Hardneck & Hardneck & IV & $\mathrm{J}$ \\
\hline W6-12840 & Italian purple artichoke & WRPIS & Softneck & Softneck & I & $\mathrm{D}$ \\
\hline W6-12842 & Poodles & WRPIS & Hardneck & Hardneck & II & $\mathrm{O}$ \\
\hline W6-12844 & 890609 & WRPIS & Softneck & Softneck & I & $\mathrm{D}$ \\
\hline W6-12912 & Montana Roja & WRPIS & Hardneck & Hardneck & II & $\mathrm{F}$ \\
\hline W6-17074 & Chinese & WRPIS & Softneck & Softneck & I & $\mathrm{H}$ \\
\hline W6-17281 & W6 17281 & WRPIS & Hardneck & Hardneck & I & $\mathrm{H}$ \\
\hline W6-18723 & 1 & WRPIS & Hardneck & Hardneck & IV & $\mathrm{N}$ \\
\hline W6-18724 & 2 & WRPIS & Softneck & Softneck & I & $\mathrm{D}$ \\
\hline W6-18726 & 4 & WRPIS & Softneck & Softneck & I & D \\
\hline W6-18729 & 7 & WRPIS & Softneck & Softneck & & D \\
\hline PI 383817 & Domasen & WRPIS & Softneck & Softneck & IV & \\
\hline PI 383819 & Pristinski & WRPIS & Softneck & Silverskin & I & B \\
\hline PI 383820 & Prizrenski & WRPIS & Softneck & Softneck & I & B \\
\hline
\end{tabular}


Table 2 (continued). Names, phenotypic classification, and genetic classification of garlic clones.

\begin{tabular}{|c|c|c|c|c|c|c|}
\hline Cultivar identifier & WRPIS cultivar & Source & Type & Class & PCA & Group \\
\hline PI 383821 & Lokalen & WRPIS & Softneck & Softneck & I & $\mathrm{E}$ \\
\hline PI 383822 & Veleski & WRPIS & Softneck & Softneck & I & B \\
\hline PI 383823 & Ivankovski & WRPIS & Softneck & Softneck & I & $\mathrm{C}$ \\
\hline PI 383824 & Lokalen & WRPIS & Softneck & Softneck & I & $\mathrm{P}$ \\
\hline PI 383831 & Klisurski & WRPIS & Softneck & Softneck & I & $\mathrm{E}$ \\
\hline PI 493097 & & WRPIS & Softneck & Softneck & II & $\mathrm{O}$ \\
\hline PI 493106 & 61 & WRPIS & Softneck & Softneck & & $\mathrm{P}$ \\
\hline PI 493114 & Cinsky & WRPIS & Softneck & Softneck & I & B \\
\hline PI 493115 & Prim & WRPIS & Softneck & Silverskin & I & B \\
\hline PI 493116 & 174 & WRPIS & Hardneck & Hardneck & IV & \\
\hline PI 493117 & Tybet 1814 & WRPIS & Softneck & Softneck & & $\mathrm{C}$ \\
\hline PI 493118 & 188 & WRPIS & Softneck & Softneck & & A \\
\hline PI 493124 & Chonan & WRPIS & Hardneck & Hardneck & I & $\mathrm{C}$ \\
\hline PI 497942 & & WRPIS & Hardneck & Hardneck & & \\
\hline PI 497943 & & WRPIS & Softneck & Softneck & I & $\mathrm{C}$ \\
\hline PI 497944 & & WRPIS & Hardneck & Hardneck & II & $\mathrm{G}$ \\
\hline PI 497947 & & WRPIS & Softneck & Softneck & I & $\mathrm{B}$ \\
\hline PI 515972 & Music (rocambole type) & WRPIS & Hardneck & Rocambole & II & \\
\hline PI 540314 & longicuspis & WRPIS & Hardneck & longicuspis & & Q \\
\hline PI 540327 & Mexicano-B & WRPIS & Hardneck & Hardneck & II & $\mathrm{O}$ \\
\hline PI 540331 & Horsky & WRPIS & Hardneck & Hardneck & & A \\
\hline PI 540333 & Duggnskij-Mestnyj & WRPIS & Hardneck & Hardneck & II & $\mathrm{F}$ \\
\hline PI 540334 & Monsanskij & WRPIS & Hardneck & Hardneck & IV & $\mathrm{J}$ \\
\hline PI 540335 & Americky Maly & WRPIS & Hardneck & Hardneck & IV & $\mathrm{J}$ \\
\hline PI 540336 & Seversky Palicak & WRPIS & Hardneck & Hardneck & IV & \\
\hline PI 540337 & Adizanskij & WRPIS & Hardneck & Hardneck & & \\
\hline PI 540338 & Starobelski belyj & WRPIS & Hardneck & Hardneck & IV & $\mathrm{L}$ \\
\hline PI 540340 & Jampol skij & WRPIS & Hardneck & Hardneck & III & I \\
\hline PI 540343 & W6 4177 & WRPIS & Hardneck & Hardneck & II & $\mathrm{F}$ \\
\hline PI 540344 & W6-4178 & WRPIS & Hardneck & Hardneck & II & $\mathrm{G}$ \\
\hline PI 540346 & W6-4180 & WRPIS & Hardneck & Hardneck & II & \\
\hline PI 540351 & W6-4185 & WRPIS & Hardneck & Hardneck & II & G \\
\hline PI 540352 & W6-4186 & WRPIS & Hardneck & Hardneck & II & G \\
\hline PI 540353 & W6 4187 & WRPIS & Hardneck & Hardneck & II & $\mathrm{F}$ \\
\hline PI 540356 & W6-4190 & WRPIS & Hardneck & Hardneck & IV & \\
\hline PI 540357 & longicuspis & WRPIS & Hardneck & longicuspis & IV & $\mathrm{L}$ \\
\hline PI 540359 & Rocambole & WRPIS & Softneck & Softneck & I & \\
\hline PI 540360 & Spanish Red & WRPIS & Hardneck & Rocambole & II & $\mathrm{F}$ \\
\hline PI 540361 & De vivo & WRPIS & Hardneck & Rocambole & II & $\mathrm{F}$ \\
\hline PI 540363 & 850904-09 & WRPIS & Hardneck & Hardneck & II & G \\
\hline PI 540365 & Early Red & WRPIS & Hardneck & Hardneck & II & $\mathrm{F}$ \\
\hline PI 540369 & Creole & WRPIS & Hardneck & Softneck & I & $\mathrm{B}$ \\
\hline PI 540370 & S\&H Italian purple & WRPIS & Softneck & Softneck & I & $\mathrm{B}$ \\
\hline PI 543048 & WKP-88-6 & WRPIS & Softneck & Softneck & I & $\mathrm{B}$ \\
\hline PI 543049 & WKP-99-19 & WRPIS & Hardneck & Hardneck & & \\
\hline PI 576914 & longicuspis & WRPIS & Hardneck & longicuspis & IV & $\mathrm{K}$ \\
\hline PI 615417 & Gomecari & WRPIS & Softneck & Artichoke & I & $\mathrm{E}$ \\
\hline PI 615418 & Achaiami & WRPIS & Hardneck & Hardneck & II & $\mathrm{F}$ \\
\hline PI 615419 & W6-4264 & WRPIS & Hardneck & Hardneck & IV & $\mathrm{J}$ \\
\hline PI 615422 & Blanco de huelma zamora & WRPIS & Softneck & Softneck & I & $\mathrm{E}$ \\
\hline PI 615423 & Blanco de huelma zamora & WRPIS & Softneck & Softneck & I & $\mathrm{E}$ \\
\hline PI 615424 & Fino de chincko ajofrin & WRPIS & Softneck & Softneck & I & B \\
\hline PI 615425 & B92-18 & WRPIS & Softneck & Softneck & I & $\mathrm{E}$ \\
\hline Ajo Rojo & & Commercial & Hardneck & Creole & I & $\mathrm{R}$ \\
\hline Allium sp. & & WRPIS & Hardneck & & & \\
\hline Bogatyr-1 & & Commercial & Hardneck & Marble PS & IV & $\mathrm{J}$ \\
\hline Bogatyr-2 & & Commercial & Hardneck & Marble PS & IV & $\mathrm{J}$ \\
\hline Bogatyr-3 & & Commercial & Hardneck & Marble PS & IV & \\
\hline Bogatyr-4 & & Commercial & Hardneck & Marble PS & & \\
\hline
\end{tabular}


Table 2 (continued). Names, phenotypic classification, and genetic classification of garlic clones.

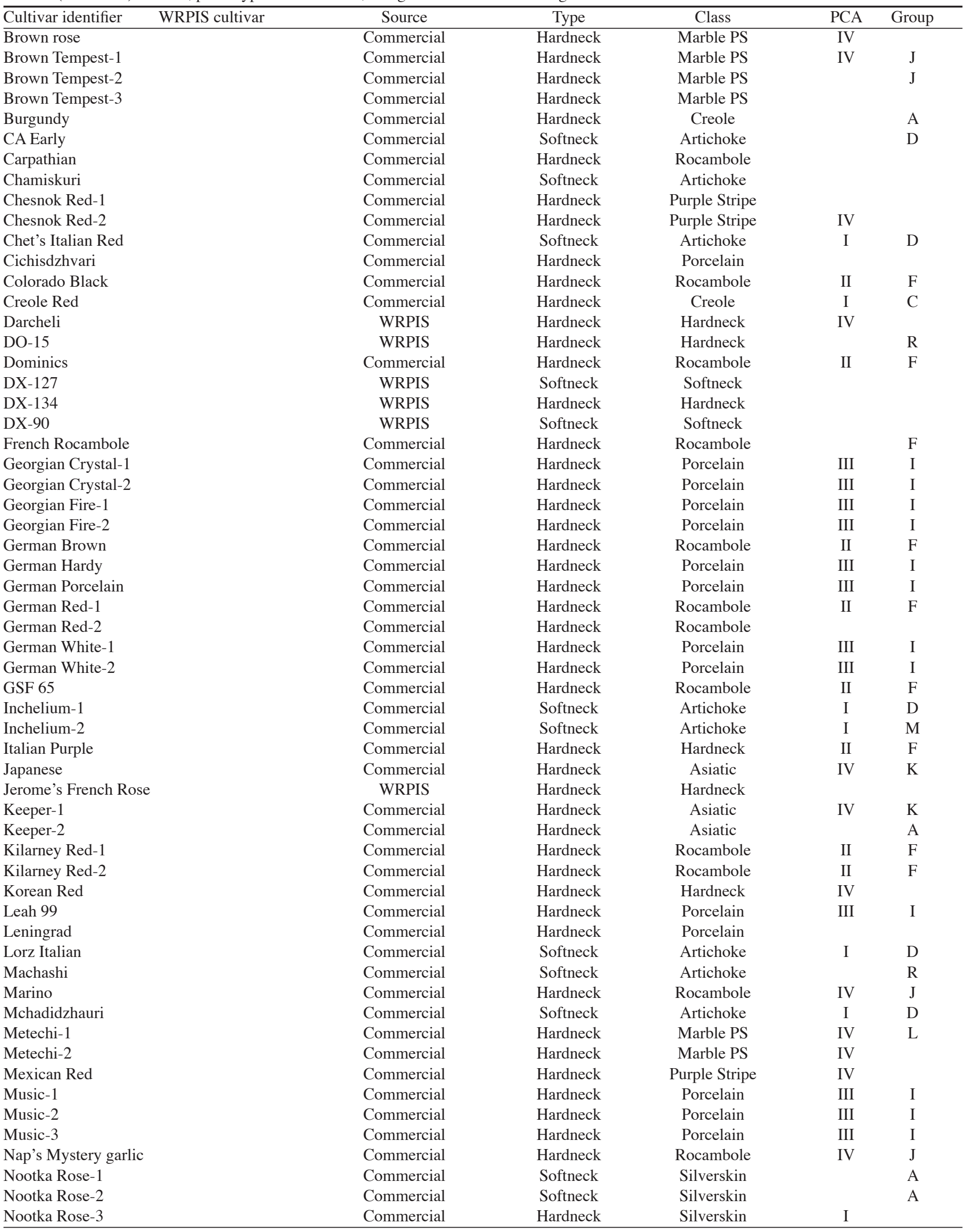


Table 2 (continued). Names, phenotypic classification, and genetic classification of garlic clones.

\begin{tabular}{|c|c|c|c|c|c|}
\hline Cultivar identifier & Source & Type & Class & PCA & Group \\
\hline Northern White & Commercial & Hardneck & Porcelain & III & $\mathrm{I}$ \\
\hline Oregon Blue & Commercial & Softneck & Artichoke & I & $\mathrm{D}$ \\
\hline Orting & WRPIS & Hardneck & Hardneck & & \\
\hline Persian Star-1 & Commercial & Hardneck & Purple Stripe & IV & $\mathrm{L}$ \\
\hline Persian Star-2 & Commercial & Hardneck & Purple Stripe & IV & $\mathrm{L}$ \\
\hline Polish Hardneck-1 & Commercial & Hardneck & Porcelain & III & I \\
\hline Polish Hardneck-2 & Commercial & Hardneck & Porcelain & III & I \\
\hline Pskem & Commercial & Hardneck & Marble PS & & \\
\hline Purple glazer & Commercial & Hardneck & Glazed PS & IV & $\mathrm{N}$ \\
\hline Purple tip & Commercial & Hardneck & Hardneck & & \\
\hline Pyong Vang Korean & Commercial & Hardneck & Asiatic & IV & K \\
\hline Randl Colorado White & Commercial & Softneck & Softneck & I & $\mathrm{D}$ \\
\hline Red Italian-1 & Commercial & Softneck & Softneck & I & $\mathrm{D}$ \\
\hline Red Italian-2 & Commercial & Softneck & Artichoke & & $\mathrm{D}$ \\
\hline Red Janice-1 & Commercial & Hardneck & Turban & I & $\mathrm{H}$ \\
\hline Red Janice-2 & Commercial & Hardneck & Turban & & \\
\hline Red Toch & Commercial & Softneck & Artichoke & I & M \\
\hline Red/Purple Italian & Commercial & Softneck & Artichoke & I & $\mathrm{D}$ \\
\hline Romanian Red & Commercial & Hardneck & Porcelain & III & I \\
\hline Russian Red-1 & Commercial & Hardneck & Rocambole & II & $\mathrm{F}$ \\
\hline Russian Red-2 & Commercial & Hardneck & Rocambole & II & $\mathrm{F}$ \\
\hline Russian Red-3 & Commercial & Hardneck & Rocambole & II & $\mathrm{F}$ \\
\hline Samarkand & Commercial & Hardneck & Hardneck & IV & $\mathrm{L}$ \\
\hline Siberian-1 & Commercial & Hardneck & Marble PS & IV & \\
\hline Siberian-2 & Commercial & Hardneck & Marble PS & IV & $\mathrm{J}$ \\
\hline Silverwhite & Commercial & Softneck & Silverskin & I & $\mathrm{C}$ \\
\hline Sonora & Commercial & & & & $\mathrm{H}$ \\
\hline Spanish Roja-1 & Commercial & Hardneck & Rocambole & II & $\mathrm{F}$ \\
\hline Spanish Roja-2 & Commercial & Hardneck & Rocambole & & $\mathrm{F}$ \\
\hline Spanish Roja-3 & Commercial & Hardneck & Rocambole & II & $\mathrm{F}$ \\
\hline Susanville & Commercial & Softneck & Artichoke & I & $\mathrm{D}$ \\
\hline Vladivostok \#1 & WRPIS & Hardneck & Hardneck & I & \\
\hline Western Rojo & Commercial & Hardneck & Hardneck & & $\mathrm{F}$ \\
\hline Yugo Red & Commercial & Hardneck & Rocambole & II & $\mathrm{F}$ \\
\hline
\end{tabular}

Table 3. AFLP primers and number of bands scored for each selective primer.

\begin{tabular}{|c|c|c|}
\hline \multicolumn{3}{|l|}{ Adapters } \\
\hline EcoRI adapter & 5'-CTC GTA GAC TGC GTA CC CAT CTG ACG GAT GGT TAA-5' & \\
\hline MseI adapter & 5'GAC GAT GAG TCC TGA GTA CTC AGG ACT CAT-5' & \\
\hline \multicolumn{3}{|l|}{ Pre-amplification primers } \\
\hline EcoRI & 5'-GAC TGC GTA CCA ATT CA & \\
\hline \multirow[t]{2}{*}{ MseI } & $5^{\prime}$-GAT GAG TCC TGA GTA AC & \\
\hline & & Bands \\
\hline Selective amplification primer sets & scored (no.) & \\
\hline \multirow[t]{2}{*}{ A41 } & 5'-IR-GAC TGC GTA CCA ATT CAC T & 3 \\
\hline & 5'-GAT GAG TCC TGA GTA ACT AA & \\
\hline \multirow[t]{2}{*}{ A42 } & 5'-IR-GAC TGC GTA CCA ATT CAC T & 5 \\
\hline & $5^{\prime}$-GAT GAG TCC TGA GTA ACT AG & \\
\hline \multirow[t]{2}{*}{ B21 } & 5'-IR-GAC TGC GTA CCA ATT CAC G & 6 \\
\hline & $5^{\prime}-$ GAT GAG TCC TGA GTA ACA AG & \\
\hline \multirow[t]{2}{*}{$\mathrm{C} 11$} & 5'-IR-GAC TGC GTA CCA ATT CAG C & 7 \\
\hline & $5^{\prime}-$ GAT GAG TCC TGA GTA ACA CG & \\
\hline \multirow[t]{2}{*}{ E21 } & $5^{\prime}-I R-G A C$ TGC GTA CCA ATT CAC C & 6 \\
\hline & $5^{\prime}$-GAT GAG TCC TGA GTA ACA AG & \\
\hline Total & & 27 \\
\hline
\end{tabular}




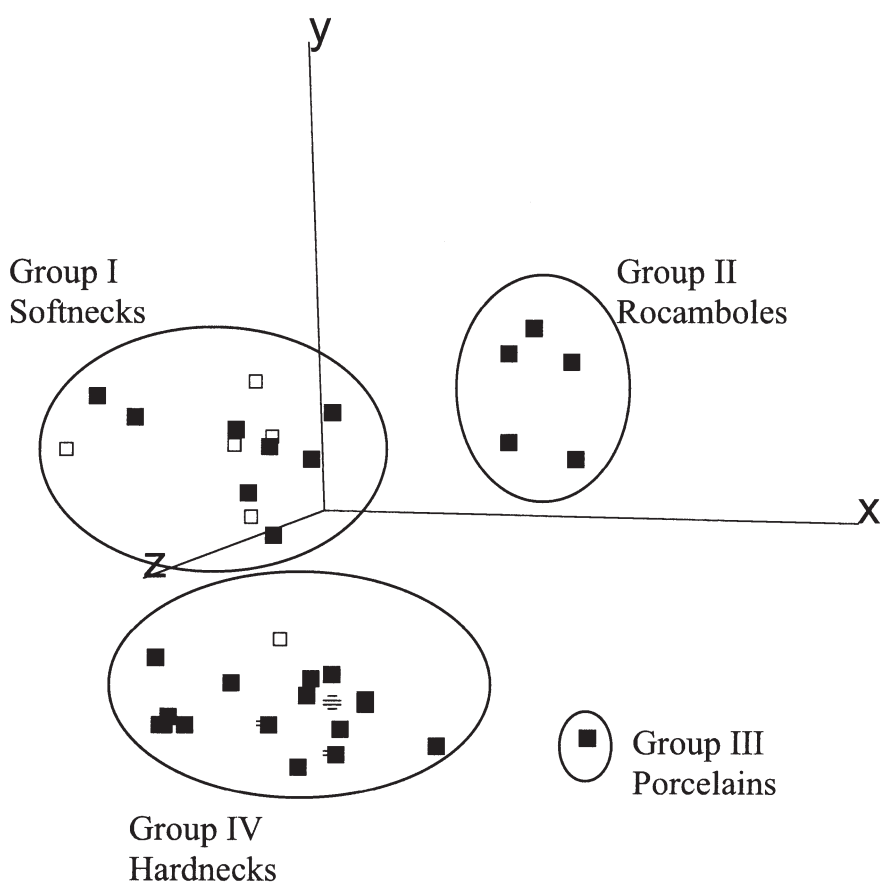

Fig. 1 (above). Principle component analysis of 158 garlic clones. Hardneck and softneck $(\square)$ types are identified. component analyses (PCA) to look for broad patterns of differentiation between hardneck and softneck classes. We used maximum parsimony analysis with boot strapped support values to refine the clustering and finally produced a minimum spanning network to show the general pattern across all 211 accessions. Networks are appropriate for intraspecific data because they are better able to represent alternate tree topologies (depicted as reticulations within the network) especially where the number of samples is large and the distances between individuals are small.

For construction of a minimum spanning network, AFLP fingerprints of all 211 accessions were merged into groups of individuals that had Euclidean distances of zero using MacClade 4.0 (Sinauer Associates, Mass.). Each group, and single accessions that did not group together, were plotted as nodes in a minimum spanning network using the computer algorithm Minspnet (Excoffier et al., 1992, http://cmpg.unibe.ch/services/software.htm). The minimum spanning tree was manually drawn using computer outputs. The number of accessions grouped within a node is reflected in the diameter of the node.

Fig. 2 (below). Minimum spanning distance diagram illustrates the genetic relatedness among 211 garlic clones. Node size represents the number of genetically identical accessions within a lettered node (key to letters is provided in Table 4). Accessions and nodes that are primarily softneck (open circles) or primarily hardneck (filled circle) are identified. The central region (shaded circles) is comprised of multiple accessions with many reticulations between them.

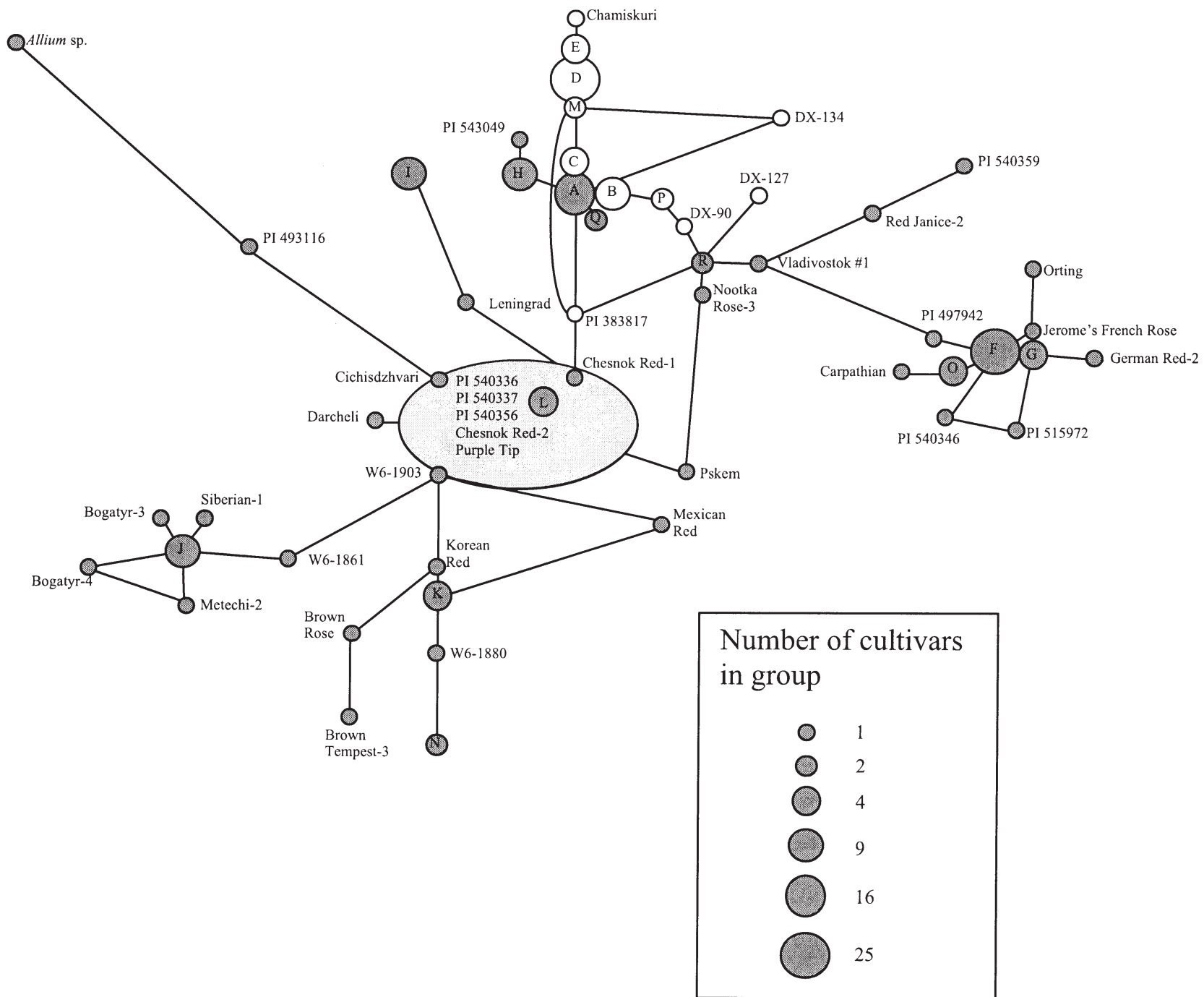


In addition, we investigated the effect of adding additional AFLP loci to differentiate accessions that had been merged together as a single group or node in the minimum spanning network. Using a smaller subset allowed us to increase the number of consistent AFLP characters and compare the genetic similarity among these accessions at higher genetic resolution. We sampled 34 accessions from four groups and compared their genotypes at 80 additional AFLP markers.

Each individual fingerprint was represented by an array of presence/absence scores for the 27 characters. A pairwise distance matrix using Jacquard's distance coefficient (using presence data only) was generated in NTSyspc ver. 2.1 (Exceter Software, N.Y.) using the set of 158 samples with complete genotypes. Principle component analyses were performed using the first three eigenvectors of the distance matrix. Parsimony analysis for the set of 158 complete genotypes was conducted with PAUP 4.0 (Sinauer Associates). Statistical support for parsimony clustering was obtained using 1000 bootstrap replications using one tree per replication. Analysis of molecular variance (AMOVA) among clades identified in parsimony was conducted using Arlequin v. 2.0 (Schneider et al., 2000).

\section{Results}

Of the 333 pairs of original replicate samples, 122 pairs of replicates were discarded from any analyses since a consensus score could not be determined. The remaining 211 samples were included in the minimum spanning distance analyses. Of those 211 samples, 158 samples had no missing data. These samples were used for parsimony, PCA, and AMOVA. Table 2 lists the garlic accessions that were included in the analyses.

Principle component analyses were performed for the 158 accessions that contained no missing data. The first, second, and third principle components accounted for $37 \%, 21 \%$, and $12 \%$, respectively, of the total variation and were plotted (Fig. 1). Softneck and hardneck garlic types can be distinguished (Group

Table 4. Groups of identical accessions as identified by MinSpnet.

\begin{tabular}{|c|c|c|c|c|}
\hline$\overline{\mathbf{A}}$ & D (continued) & F Rocambole & $\mathbf{H}$ & K Asiatic \\
\hline Burgundy & Oregon Blue & Colorado Black & Red Janice-1 & Japanese \\
\hline Keeper-2 & Randl Colorado White & Dominics & Sonora & Keeper-1 \\
\hline Nootka Rose-1 & Red Italian-1 & French Rocambole & W6-1862 & Pyong Vang Korean \\
\hline Nootka Rose-2 & Red Italian-2 & German Brown & W6-1883 & PI 576914 \\
\hline W6-49 & Red/Purple Italian & German Red-1 & W6-10473 & \\
\hline W6-8413 & Susanville & GSF 65 & W6-17074 & L Purple Stripe \\
\hline W6-8415 & W6-671 & Italian Purple & W6-17281 & Metechi-1 \\
\hline W6-8417 & W6-672 & Kilarney Red-1 & & Persian Star-1 \\
\hline W6-8420 & W6-2560 & Kilarney Red-2 & I Porcelain & Persian Star-2 \\
\hline W6-12830 & W6-2561 & Russian Red-1 & GA Crystal-1 & Samarkand \\
\hline W6-12831 & W6-2562 & Russian Red-2 & GA Crystal-2 & W6-1885 \\
\hline W6-12832 & W6-2563 & Russian Red-3 & GA Fire-1 & PI 540338 \\
\hline PI 493118 & W6-8403 & Spanish Roja-1 & GA Fire-2 & PI 540357 \\
\hline \multirow[t]{2}{*}{ PI 540331} & W6-8404 & Spanish Roja-2 & German Hardy & \\
\hline & W6-8405 & Spanish Roja-3 & German Porcelain & $\mathbf{M}$ \\
\hline B & W6-8406 & Western rojo & German White-1 & Inchelium-2 \\
\hline PI 383819 & W6-8409 & Yugo Red & German White-2 & Red Toch \\
\hline PI 383820 & W6-8410 & W6-12824 & Leah 99 & \\
\hline PI 383822 & W6-8411 & W6-12825 & Music-1 & $\mathbf{N}$ \\
\hline PI 493114 & W6-10729 & W6-12828 & Music-2 & Purple glazer \\
\hline PI 493115 & W6-10734 & W6-12829 & Music-3 & W6-18723 \\
\hline PI 497947 & W6-10736 & W6-12833 & Northern White & \\
\hline PI 540369 & W6-10737 & W6-12837 & Polish Hardneck-1 & $\mathbf{O}$ \\
\hline PI 540370 & W6-10739 & W6-12838 & Polish Hardneck-2 & W6-12823 \\
\hline PI 543048 & W6-12816 & W6-12912 & Romanian Red & W6-12842 \\
\hline \multirow[t]{2}{*}{ PI 615424} & W6-12836 & PI 540333 & PI 540340 & PI 493097 \\
\hline & W6-12840 & PI 540343 & & PI 540327 \\
\hline $\mathbf{C}$ & W6-12844 & PI 540353 & J Marble Purple Stripe & \\
\hline Silverwhite & W6-18724 & PI 540360 & Bogatyr-1 & $\mathbf{P}$ \\
\hline PI 383823 & W6-18726 & PI 540361 & Bogatyr-2 & PI 383824 \\
\hline PI 493117 & W6-18729 & PI 540365 & Brown Tempest-1 & PI 493106 \\
\hline PI 493124 & & PI 615418 & Brown Tempest-2 & \\
\hline PI 497943 & $\mathbf{E}$ & & Marino & $\mathbf{Q}$ \\
\hline \multirow{2}{*}{ Creole Red } & PI 383821 & G Rocambole & Nap's Mystery garlic & W6-8414 \\
\hline & PI 383831 & PI 497944 & Siberian-2 & W6-8416 \\
\hline D Artichoke & PI 615417 & PI 540344 & W6-12839 & PI 540314 \\
\hline CA Early & PI 615422 & PI 540351 & PI 540334 & \\
\hline Chet's Italian Red & PI 615423 & PI 540352 & PI 540335 & $\mathbf{R}$ \\
\hline Inchelium-1 & PI 615425 & PI 540363 & PI 615419 & Ajo Rojo \\
\hline Lorz Italian & & & & Machashi \\
\hline Mchadidzhauri & & & & DO-1 \\
\hline
\end{tabular}




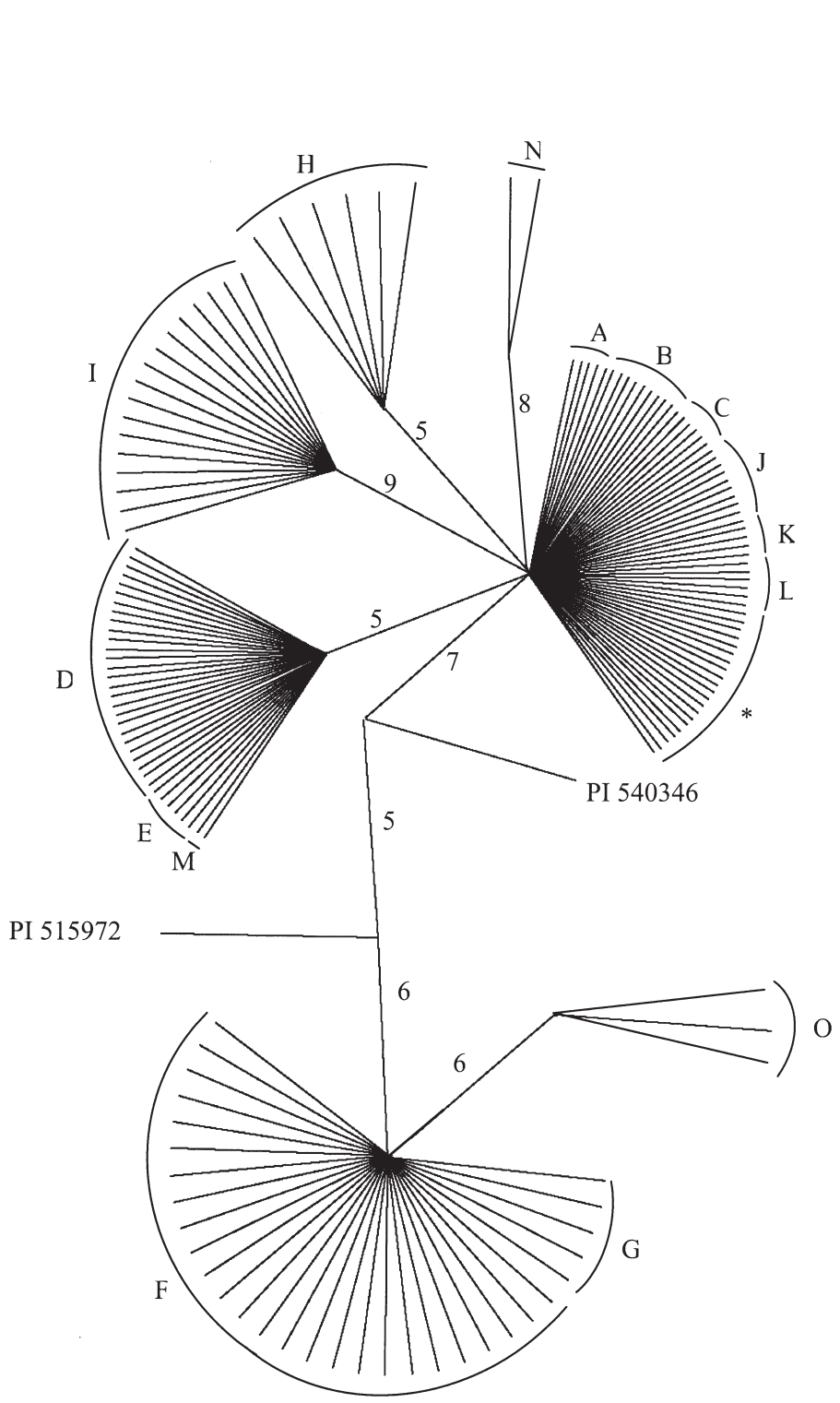

Fig. 3. Parsimony analysis ( 1000 bootstrap replicates) of 158 garlic clones. Letters refer to groups of genetically identical accessions defined in Table 4.

I vs. Groups II, III, and IV). Clusters of rocambole (Group II) and porcelain (Group III) accessions can be identified within the hardneck grouping. Accessions in each cluster are identified in Table 2. Softneck garlic types represent less genetic diversity than the hardneck types (Fig. 1) since they tend to be restricted to one cluster in principle component space.

The minimum spanning tree (Fig. 2) shows structure among the complete set of 211 accessions. An accession of Allium sp. (W6-12827) was included as the outgroup in the minimum spanning tree. Scape formation and classification data were overlaid on the minimum spanning tree to determine if phenotypes could be correlated with genetic distances. Many of the regions of the minimum spanning tree could be assigned to the grower classification categories. Our ability to assign phenotypic categories to all the samples included in the network is limited since most of the NPGS garlic collection have not been classified in this manner. The major classification groups of artichoke, rocambole,
* $\quad$ porcelain, purple stripe, marble purple stripe, $\mathrm{P} \quad$ and Asiatic each included a set of genetically Q identical accessions (Table 4). In most cases, $\mathrm{R} \quad$ all the accessions within a group of genetically Ajo Rojo Brown Rose Bogatyr-3 Chesnok Red-2 Darcheli Korean Red-3 Metechi-2 Mexican Red Nootka Rose-3 Siberian-1 Vladivostok \#1 PI 383817 PI 493116 PI 540356 PI 540359 W6-1903 W6-1861 identical accessions belonged to the same phenotypic classification category. However, rocambole types 'Marino’ and 'Nap's Mystery Garlic' clustered with the marble purple stripes (Tables 2 and 4). The most reticulations in the network were displayed in the purple stripe region $(\mathrm{L})$. This region also is the basal region of the network as it shared the most identity with the out-group. To simplify the display, the nodes within this region were grouped in an ellipse with the component accessions listed. Overall, we identified 18 groups of genetically identical accessions using our 27 AFLP markers (Table 4). Replicate accessions of 'Keeper' and 'Metechi' did not cluster together in our analyses.

Using parsimony analysis on the set of 158 complete genotypes, we tested some of the statistical support for the structure revealed in the minimum spanning tree. The topology of the parsimony tree, while statistically supporting genetic differentiation among some accessions of marble purple stripes, porcelains, artichokes, and rocamboles, collapsed many accessions into large clades (Fig. 3). This condensing of data is not unexpected in this type of phylogenetic analysis given the structure of the number of accessions (158) and the number of informative characters (27) and underscores the uncertainty of the phylogenetic history of these clonal lineages using these 27 loci. Despite this, the parsimony and genetic distance based approaches revealed many common attributes in the diversity among these accessions.

While our phylogenetic analysis of the genetic relationships of U.S. garlic varieties suggests that phenotypic categories correlate with genetic distance data, some categories, such as turbans and creoles, require additional examination. We also believe that some diverse accessions may not be easily classified directly into the current commercial categories. For instance, A. longicuspis and creole types do not show any single genetic identity to other groups in the data set. We used AMOVA techniques to generate pairwise $\mathrm{F}_{\mathrm{st}}$ to measure genetic differentiation between accessions in nine phenotypic classes listed in Table 2. These accessions include artichoke, asiatic, creole, $A$. longicuspis, marble purple stripe, porcelain, purple stripe, rocambole, and silverskin. The significance of these $\mathrm{F}_{\mathrm{st}}$ estimates was tested using a permutation algorithm in Arlequin. The results showed that while seven of the nine groups were significantly differentiated, creole types and A. longicuspis were not (data not shown). Creole and A. longicuspis classification types may not be robust and may not reflect their true taxonomic origin. Of the 118 NPGS accessions, 24 had unique genotypes and of the 75 commercial accessions, 19 had unique genotypes. Overall $64 \%$ of the WRPIS and $41 \%$ of the commercial accessions were duplicates as determined by this method. 
Table 5. 107 loci were scored on the groups of accessions that were identified based on 27 loci.

\begin{tabular}{lccc}
\hline Gnique & $\begin{array}{c}\text { Loci } \\
\text { cultivars } \\
\text { (no.) }\end{array}$ & $\begin{array}{c}\text { scored } \\
\text { (no.) }\end{array}$ & $\begin{array}{c}\text { Identity } \\
(\%)\end{array}$ \\
\hline D & 9 & 107 & 99.6 \\
F & 10 & 107 & 99.1 \\
I & 10 & 107 & 99.7 \\
J & 5 & 107 & 100.0 \\
\hline
\end{tabular}

\section{Discussion}

Genetic analysis using AFLP in garlic was a challenging endeavor. Garlic has an unusually large genome size of $>3 \times$ $10^{10}$ basepairs (Ranjekar et al., 1978) and even though the selective primer used four additional random bases for refining the selection of amplification products, comparisons made across hundreds of individuals resulted in reduced accuracy due to amplification instability. To solve this, we reduced the number of scorable markers to a verifiable subset and tested each of these markers for accuracy by genotyping two independent genomic DNA extractions from each accession.

We scored an additional 80 bands on 34 duplicate accessions to determine if the inclusion of additional loci would differentiate the samples that we identified as duplicates. As shown in Table 5 , we had $>99 \%$ identity among consensus scores for samples that were deemed identical based on 27 loci. Ipek et al (2003) initially scored their accession data for 80 loci, and then increased the number of loci to 183 to determine their confidence in their assessment of duplication. Ipek et al (2003) were able to differentiate additional clones as they increased the number of scored loci in their dataset. These authors pooled multiple clones of a given accession for their analyses, but there is no indication that replicate samples were analyzed. Bradley et al. (1996) also identified uniquely named accessions with identical genotypes.

Matus et al. (1999) measured 17 phenological characters of 66 garlic clones and performed PCA to classify these accessions. These authors plotted the first and third principle components and could distinguish clones with flower stalks from those with incomplete and nonflowering phenotypes. Using RAPD analyses, Bradley et al. (1996) also separated flowering and nonflowering or incomplete flowering garlic accessions.

Our PCA analysis distinguished between many of the hardneck and softneck garlic types. We note that hardneck varieties that group with softneck varieties in our PCA have similar clustering patterns in the network analysis. Similar trends were identified by Maass and Klaas (1995), who used neighbor joining analyses to analyze isozyme data for 300 garlic clones. They could differentiate early senescing, nonfertile flowering clones from softneck clones from A. longicuspis clones collected from central Asia. Maass and Klaas (1995) used RAPD analyses to further distinguish softneck types from the Caucasus and Mediterranean regions (incomplete bolting artichoke types) from softneck types from east and middle Asia. Al Zahim et al. (1997) correlated morphological variation with RAPD patterns and could differentiate rocamboles and most softnecks from other hardnecks.

We chose to present our genetic distance data as a minimum spanning tree, rather than a dendogram. This method creates the smallest network length based on genetic distance values and several equally short networks are shown as reticulations in the network where a node has more than two connections. It also presents identical accessions as nodes within the tree, which we felt was suitable since there were many examples of duplicate accessions within our dataset.

Since garlic phenotypes are known to vary according to environmental conditions, the descriptions of the classes of garlic in Table 1 are only relevant in the growing conditions in Okanogan, Wash. Most of the commercial clones of garlic have been classified according to their phenotypes at this location. Clones that fall within a given category are phenotypically similar. In most cases, clones that are phenotypically similar are also genotypically similar, as determined by our molecular analyses. Additional studies are needed to determine which phenotypic traits can accurately describe the phenotypic categories regardless of location.

Our data identify distinct groups of artichoke, rocambole, marble purple stripe, asiatic, porcelain and purple stripe garlics. There are some hardneck accessions that do not genetically align with the described hardneck categories. Many of these are NPGS accessions that are phenotypically unclassified.

Our data place the Asiatic type within the hardnecks, as opposed to the softneck category where it was classified by Pooler and Simon (1993). Our genetic results place one replicate of 'Keeper', phenotypically an Asiatic, within the softneck group. 'Keeper', a hardneck, was isolated after multiple selections from a population of softnecks (W. Lyons, personal communication), so it is not surprising that it is genetically more similar to softnecks than hardnecks. We have identified some phenotypic Asiatics that do not align with the other Asiatics. Perhaps the Asiatic phenotype extends to a number of genetically distinct accessions.

Our study included only a few turban and creole garlic types. Since the number of accessions is limited, and phenotypically similar accessions do not align, we can not speculate on the genetic relationships between and among turban and creole accession types.

Al-Zahim et al. (1997), determined that the clone 'Burgundy' (type creole) clusters with nonbolting-type garlic clones by RAPD analysis, even though it has been classified as a boltingtype garlic. Similar results were reported by Maass and Klaas (1995). Our studies also suggest that 'Burgundy' clusters with nonbolting types of garlic.

Ipek et al. (2003) and Pooler and Simon (1993) have generated dendograms for 48 and 100 garlic accessions, respectively, based on isozyme, RAPD, and AFLP data. Nine accessions were common between our dataset and the phylogeny generated by Ipek et al. (2003). There is some agreement between the placements by Ipek and the placements reported in this study. Softneck accessions PI 383823, PI 383819, PI 497943, and PI 383824 colocalized with softneck types in both studies. Hardneck accession PI 493116 localized with other hardnecks in both studies.

Our phylogeny suggests that A. longicuspis is indistinguishable from A. sativum, as was found by Pooler and Simon (1993), Ipek et al. (2003), and Al-Zahim et al. (1997). Ipek et al. (2003) and Pooler and Simon (1993) were unable to correlate the geographical origin of their samples to phylogenetic relationships. Many of the garlic accessions in the U.S. collection may have secondary source data, rather than original wild collection data associated with them. Due to the difficulty of tracing garlic clones to geographical origins and the lack of correlation between banding patterns and geographical origin, we did not perform any geographic analyses.

This study determined that the uniquely named NPGS and commercial collections are $64 \%$ and $41 \%$ duplicate accessions, respectively. Unique commercial and NPGS accessions were 
identified. When NPGS accessions are classified according to phenotype using a scheme similar to the one proposed by Engeland $(1991,1995)$ it would be useful to determine if these classifications are supported by our genetic analyses. Seventy-nine NPGS accessions were not included in these studies since we could not generate a consensus score with $<4 \%$ error rate. We can prioritize the genotyped portion of the NPGS garlic collection for cryopreservation by assuming that a single accession representing each genetic group of accessions as well as the genetically unique accessions should have priority over duplicate samples.

\section{Literature Cited}

Al-Zahim, H.J. Newbury, and B.V. Ford-Lloyd. 1997. Classification of genetic variation in garlic (Allium sativum L.) revealed by RAPD. HortScience 32:1102-1104.

Bradley, K.F., M.A. Rieger, and G.G. Collins. 1996. Classification of Australian garlic cultivars by DNA fingerprinting. Aust. J. Expt. Agr. 36:613-618.

Cho, E., H. Kim, J. Kim, H. Baek, C. Kim, and F. Englemann. 2003. Proceedings of the international workshop on cryopreservation of bio-genetic resources. Rural development administration, Suwon, Republic of Korea.

Engeland, R.L. 1991. Growing great garlic. Filaree Productions, Okanogan, Wash.

Engeland, R.L. 1995. Growing great garlic (suppl.). Filaree Productions, Okanogan, Wash.

Etoh, T. and P.W. Simon. 2002. Diversity, fertility and seed production of garlic, p. 101-117. In: H.D. Rabinowitch and L. Currah (ed.). Allium crop science: Recent advances. CAB Intl.

Excoffier, L., P.E. Smouse, and J.M. Quattro. 1992. Analysis of molecular variance inferred from metric distances among DNA haplotypes: Application to human mitochondrial DNA restriction data. Genetics 131:479-491.
Hanelt,P. 2001. Mansfeld's encyclopedia of agricultural and horticultural crops (except ornamental). Springer, Berlin.

Ipek, M., A. Ipek, and P.W. Simon. 2003. Comparison of AFLPs, RAPD markers, and isozymes for diversity assessment of garlic and detection of putative duplicates in germplasm collections. J. Amer. Soc. Hort. Sci. 128:246-252.

Keller, E.R.J. 2002. Cryopreservation of Allium sativum L. (garlic), p. 37-47. In: L.E. Towill and Y.P.S. Bajaj (eds.). Biotechnology in agriculture and forestry, cryopreservation of plant germplasm II. Springer-Verlag, Berlin.

Maass, H.I. and M. Klaas. 1995. Infraspecific differentiation on garlic (Allium sativum L.) by isozyme and RAPD markers. Theor. Appl. Genet. 91:89-97.

Makowska, Z., J. Keller, and F. Engelmann. 1999. Cryopreservation of apices isolated from garlic (Allium sativum L.) bulbils and cloves. Cryo-Letters 20:175-182.

Matus, I., M.I. Gonzalez, and A. del Pozo. 1999. Evaluation of phenotypic variation in a Chilean collection of garlic (Allium sativum L.) clones using multivariate analysis. IPGRI Plant Genetic Res. Nwslt. 117:31-36.

Myburg, A.A. and D.L. Remington. 2000. Protocol for high-throughput AFLP analysis using LI-COR IR2 automated sequencers. 26 Nov. 2001.http://www.up.ac.za/academic/fabi/eucgenomics/euc_mapping/ AFLP_protocol.pdf .

Pooler, M.R. and P.W. Simon. 1993. Characterization and classification of isozyme and morphological variation in a diverse collection of garlic clones. Euphytica 68:121-130.

Ranjekar, P. K., D. Pallotta, and J.G. Lafontaine. 1978. Analysis of plant genomes. V. Comparative study of molecular properties of DNAs of seven Allium species. Biochem. Genet. 16:957-970.

Schneider, S., D. Roessli, and L. Excoffier. 2000. Arlequin-A software for population genetics data analysis. http://anthro.unige.ch/arlequin).

Vos, P., R. Hogers, M. Bleeker, M. Reijans, T. van de Lee, M. Hornes, A. Frijters, J. Pot, J. Peleman, M. Kuiper, and M. Zabeau. 1995. AFLP: A new technique for DNA fingerprinting. Nucleic Acids Res. 23:4407-4414. 